\title{
MicroRNA-497 targets hepatoma-derived growth factor and suppresses human prostate cancer cell motility
}

\author{
DEYAO WU ${ }^{1 *}$, XIAOBING NIU ${ }^{1,2^{*}}$, HUIXING PAN ${ }^{1}$, ZICHUN ZHANG $^{1}$, \\ YUNFENG ZHOU ${ }^{1}$, PING QU ${ }^{1}$ and JIAN ZHOU ${ }^{1}$ \\ ${ }^{1}$ Department of Urology, The Fourth Affiliated Hospital of Nantong Medical College, \\ Yancheng City No. 1 People's Hospital, Yancheng, Jiangsu 224001; ${ }^{2}$ Department of Urology, \\ Huai'An First People's Hospital Affiliated to Nanjing Medical University, Huai'an, Jiangsu 223300, P.R. China
}

Received December 16, 2014; Accepted December 15, 2015

DOI: $10.3892 / \mathrm{mmr} .2016 .4756$

\begin{abstract}
MicroRNA-497 (miR-497) has been reported to be downregulated in certain types of cancer, including breast, gastric, endometrial, colorectal and prostate cancer as well as hepatocellular and nasopharyngeal carcinoma. The present study aimed to investigate the underlying mechanism of the tumor suppressor function of miR-497 in prostate cancer. Following transfection with miR-497, the DU145 and PC-3 prostate cancer cell lines were subjected to Transwell migration and invasion assays, western blot analysis and a luciferase assay. It was revealed that miRNA-497 inhibited the migration and invasion of prostate cancer cells. In addition, is was indicated that miRNA-497 directly targets hepatoma-derived growth factor (HDGF) in prostate cancer cells. These results suggested that restoration of miR-497 and the resulting downregulation of HDFG may represent a promising therapeutic strategy for prostate cancer.
\end{abstract}

\section{Introduction}

Prostate cancer is one of the most common malignancies among men and is the second leading cause of cancer-associated mortality in males (1). In 2014, prostate cancer accounted for an estimate of $27 \%$ of newly diagnosed cancer cases and $10 \%$ of cancer-associated mortalities in men in the USA (2). Since prostate cancer is organ-confined in most cases at the time-point of diagnosis, radical prostatectomy and definitive radiotherapy are the current standard treatment for the vast

Correspondence to: Dr Jian Zhou, Department of Urology, The Fourth Affiliated Hospital of Nantong Medical College, Yancheng City No. 1 People's Hospital, 15 Yuehe Road, Yancheng, Jiangsu 224001, P.R. China

E-mail: luqiang0405@163.com

*Contributed equally

Key words: prostate cancer, hepatoma-derived growth factor, microRNA-497 majority of prostate cancer cases (3); however, a sub-set of patients present with disease progression, including metastasization to other organs or tissues in the body, such as bones or bladder (4). A widely accepted treatment option for such patients is androgen-deprivation therapy, following which, however, prostate cancer recurrence in its androgen-independent or hormone-refractory type is common (5). While the accurate etiology of prostate cancer is complex, several studies have suggested that it may be associated with factors including smoking, environment, dietary habits, endocrine system, age and ethnicity (6-9). In the clinic, the prevention and treatment of prostate cancer and associated metastasis have remained challenging, as the underlying molecular mechanisms have remained to be fully elucidated (1). Therefore, it is urgent to discover novel therapeutic targets and approaches to suppress prostate cancer metastasis.

Certain microRNAs (miRs) have been found to be correlated with the clinical outcome of specific cancer types (10). miRs are a class of naturally occurring, endogenous small non-coding RNAs, with a size of 19-22 nt (11). They specifically bind the 3'-untranslated regions (3'UTR) of their target messenger RNAs (mRNAs) to induce their degradation or inhibit their translation, causing partial or total silencing of the respective protein-coding gene (12). miRs are thought to regulate two-thirds of the entire protein-encoding genes (13). The expression of miRs themselves can also be regulated similarly to that of protein-coding genes, either through genetic or epigenetic shifts. miR expression is frequently aberrant in various cancer types (14). These alterations have been shown to be accountable for almost all facets of cancer development and progression (15). Upregulated miRs in cancer may function as oncogenes by negatively regulating tumor suppressors, while, downregulated miRs may function as tumor suppressor genes in healthy cells and inhibit cancer by regulating oncogenes $(16,17)$.

miR-497 was found to be significantly downregulated in prostate cancer, and its restoration was shown to have anti-proliferative and apoptotic effects (18). Based on these findings, the present study assessed the effects of miR-497 restoration on the migration and invasion of prostate cancer cells as processes associated with metastasis, and identified the underlying molecular mechanism. Hepatoma-derived growth 
factor (HDGF), which was indicated to be a putative target of miR-497, was confirmed using a luciferase reporter assay. The present study found that miR-497 represses the migratory and invasive capacity of the DU145 and PC-3 prostate cancer cells by downregulating HDGF.

\section{Materials and methods}

Cell culture and transfection. The human prostate cancer cell lines DU145 and PC-3 were purchased from the Shanghai Institute of Biochemistry and Cell Biology (Shanghai, China) and cultured in RPMI-1640 medium supplemented with $10 \%$ heat-inactivated fetal bovine serum (FBS; Gibco, Thermo Fisher Scientific, Waltham, MA, USA) in a humidified atmosphere containing $5 \% \mathrm{CO}_{2}$ at $37^{\circ} \mathrm{C}$.

Mature miR-497 mimics (5'-CAGCAGCACACUGUG GUUUGU-3') and negative control (NC) miRNA mimics (5'-UUCUCCGAACGUGUCACGUTT-3') were designed and synthesized by GenePharma (Shanghai, China). When the density of DU145 and PC-3 cells reached 30-40\%, cells were transfected using Lipofectamine 2000 (Invitrogen; Thermo Fisher Scientific), according to the manufacturer's instructions.

Cell migration and invasion assays. The cell migration and invasion assays were performed using Transwell chambers ( $8 \mu \mathrm{m}$; Costar; Thermo Fisher Scientific). For the migration assay, $1 \times 10^{5}$ transfected cells (miR-497 mimics and NC) were placed into the upper chamber cultured in medium with $2 \%$ FBS, while $500 \mu$ l medium containing $20 \%$ FBS was added to the lower chamber. For the invasion assays, $1 \times 10^{5}$ transfected cells (miR-497 mimics and NC) cultured in medium with $2 \%$ FBS were placed into the upper chamber pre-coated with Matrigel (BD Biosciences, Franklin Lakes, NJ, USA), while $500 \mu \mathrm{l}$ medium containing $20 \%$ FBS was added to the lower chamber. Cells were incubated for $12 \mathrm{~h}$ for the migration assay and $24 \mathrm{~h}$ for the invasion assay. At the end of the experiments, the cells that had not migrated or invaded through the pores were carefully removed. The filters were then fixed with $100 \%$ methanol (Beyotime Institute of Biotechnology, Inc., Haimen, China) for $2 \mathrm{~min}$, stained in $0.5 \%$ crystal violet (Beyotime Institute of Biotechnology, Inc.) for $2 \mathrm{~min}$, rinsed in phosphate-buffered saline and then subjected to microscopic inspection (magnification, x200). Five random fields were counted per chamber using an inverted microscope (CKX41; Olympus Corp., Tokyo, Japan). Each condition was assayed in triplicate and each experiment was repeated at least three times.

Western blot analysis. Primary antibodies used in the present study, including HDGF and $\beta$-actin were products of Cell Signaling Technology, Inc. (Danvers, MA, USA). DU145 or PC-3 cells were seeded in six-well plates, and $72 \mathrm{~h}$ after transfection with miR-497 mimics or NC, cells were harvested and homogenized with radioimmunoprecipitation assay lysis buffer (Beyotime Institute of Biotechnology, Inc.). The protein concentration was measured using the bicinchoninic acid protein assay kit (Beyotime Institute of Biotechnology, Inc.). Equal amounts of protein were separated using $10 \%$ sodium dodecyl sulfate polyacrylamide electrophoresis (Beyotime Institute of Biotechnology, Inc.) gel and then transferred onto polyvinylidene difluoride membranes (Beyotime Institute of
Biotechnology, Inc.), which were blocked with 5\% non-fat dry milk for $2 \mathrm{~h}$ and incubated with primary antibodies, including mouse anti-human monoclonal HDGF (cat. no. sc-271344; Santa Cruz Biotechnology, Inc., Santa Cruz, CA, USA) and mouse anti-human monoclonal $\beta$-actin (cat. no. 3700; Cell Signaling Technology, Inc.). The membrane was washed and then incubated for $1 \mathrm{~h}$ with the corresponding horseradish peroxidase-conjugated secondary antibody (sc-2005; Santa Cruz Biotechnology, Inc., CA, USA) in Tris-buffered saline with Tween 20 (Beyotime Institute of Biotechnology, Inc.). Finally, blots were visualized using enhanced chemiluminescence solution (Pierce, Rockford, IL, USA) and images were captured using the FluorChem imaging system (Alpha Innotech, San Leandro, CA, USA).

Luciferase reporter assay. A search with TargetScan (http://www.targetscan. org) predicted HDGF as a direct target gene of miR-497. A seven-nucleotide seed match of position 37-43 of the HDGF 3'-UTR with miR-497 was revealed. In order to verify this interaction, a luciferase reporter assay was performed. Cells were plated in a 12-well plate at $~ 90 \%$ confluence and transfected with miR-497 mimics or NC using Lipofectamine 2000 (Invitrogen). For the reporter assays, stably miR-497-overexpressing cells were transiently transfected with reporter plasmids driven by a wild-type or mutated fragment from the 3'UTR of HDGF. The luciferase reporter vectors, including HDGF-3'UTR Wt and HDGF-'UTR Mut, were obtained from GenePharma Co., Ltd. (Shanghai, China). Each sample was also co-transfected with $0.05 \mu \mathrm{g}$ pRL-CMV plasmid expressing Renilla Luciferase (Promega Corp., Madison, WI, USA) as an internal control for transfection efficiency. After $48 \mathrm{~h}$ of incubation, the cells were harvested and lysed, and luciferase activity was measured using the Dual-Luciferase Reporter Assay System (Promega Corp.) according to the manufacturer's instructions. Firefly luciferase activity was normalized to Renilla luciferase activity for each transfected well. Each assay was replicated three times.

Statistical analysis. Values are expressed as the mean \pm standard deviation. Comparison between groups was performed using Student's $t$-test. All statistical analyses were performed using Stata 10.0 (College Station, Texas, USA). A double-tailed P-value of $<0.05$ was considered to indicate a statistically significant difference between values.

\section{Results}

miR-497 inhibits cell migration and invasion of prostate cancer cell lines. To determine whether miR-497 regulates human prostate cancer cell migration and invasion, the DU145 and PC-3 cells were transfected with miR-497 and NC mimics and subjected to Transwell assays. As expected, overexpression of miR-497 significantly decreased the migratory and invasive capacity of DU145 and PC-3 cells (Fig. 1). These observations indicated that miR-497 was a negative regulator of prostate cancer cell migration and invasion.

miR-497 suppresses the expression of HDGF in prostate cancer cell lines. A search with TargetScan predicted that HDGF is a direct target gene of miR-497. As illustrated in Fig. 2A, position 


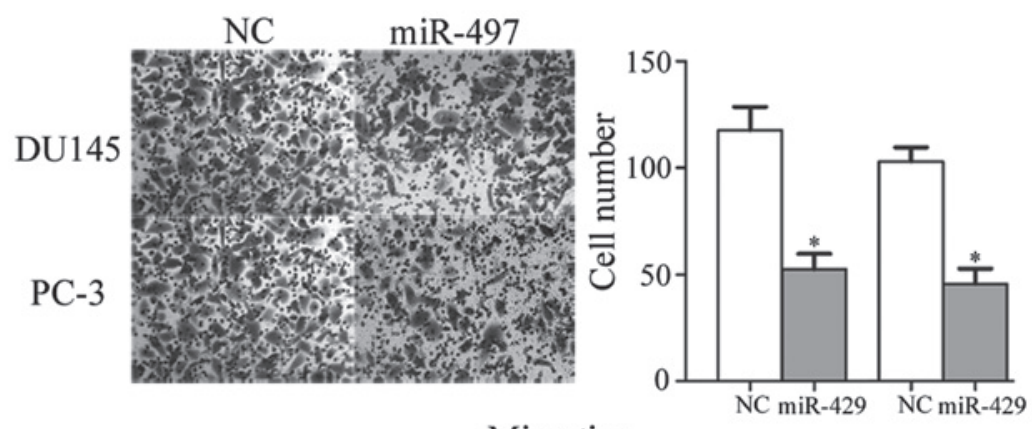

Migration

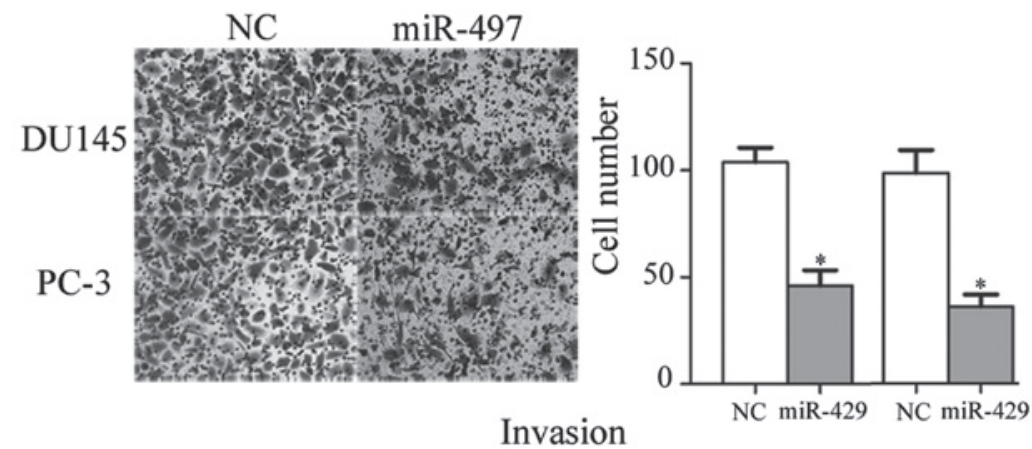

Figure 1. Inhibition of tumor cell migration and invasion by miR-497 in prostate cancer cell lines. Transwell assays showed that compared with the NC, miR-497 inhibited the migration and invasion of DU145 and PC-3 cells (magnification, $\mathrm{x} 200$ ). Values are expressed as the mean \pm standard deviation. ${ }^{*} \mathrm{P}<0.05$, compared with the control. NC, negative control; miR, microRNA.

A

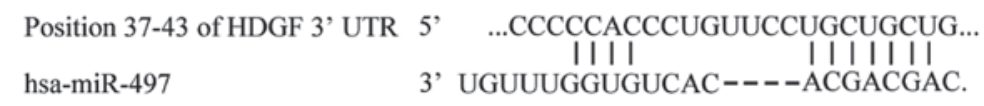

$\mathbf{B}$
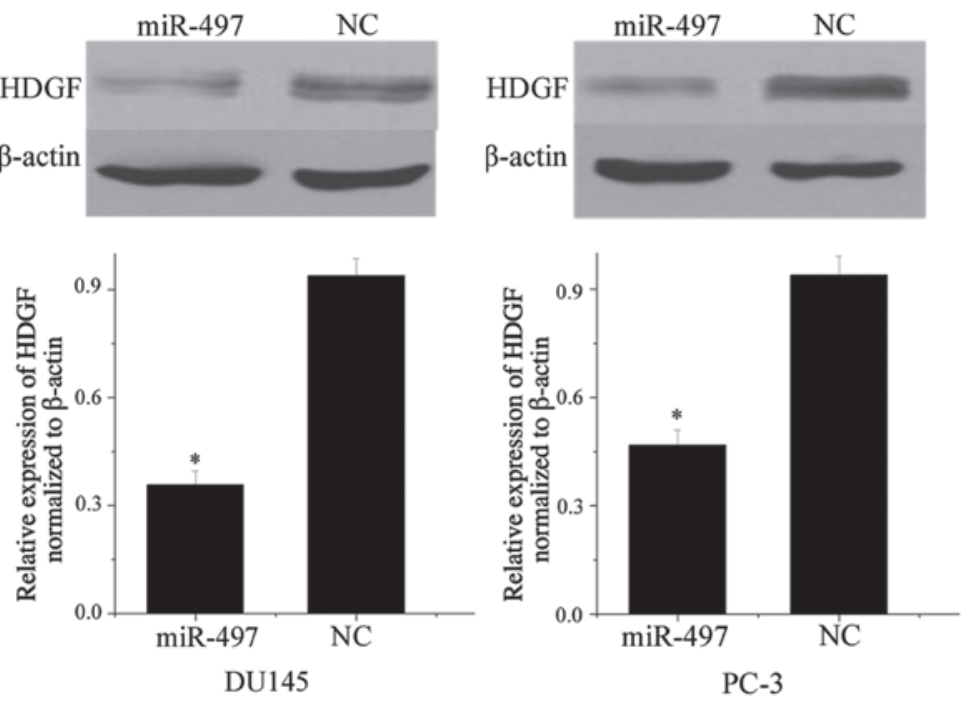

Figure 2. (A) According to bioinformatics analysis with Targetscan 5.2, the 3'UTR of HDGF mRNA contains a seven-nucleotide seed match with miR-497 at position 37-43. (B) HDGF was significantly reduced in DU145 and PC-3 cells after transfection with miR-497. Values are expressed as the mean \pm standard deviation.

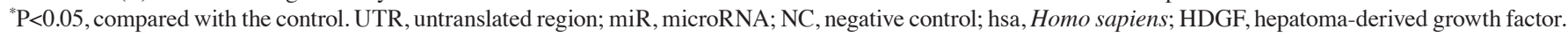

37-43 of the HDGF 3'-UTR represents a seven-nucleotide seed match with miR-497.

Western blot analysis was then performed to explore whether miR-497 affected the expression of HDGF in prostate cancer cell lines. As shown in Fig. 2B, HDGF was significantly downregulated in the DU145 and PC-3 prostate cancer cell lines after transfection with miR-497 $(\mathrm{P}<0.05)$. These results revealed that miR-497 suppressed the protein expression of HDGF in prostate cancer cell lines.

$H D G F$ is a direct target gene of miR-497 in prostate cancer. To further confirm that HDGF is a direct target of miR-497, DU145 

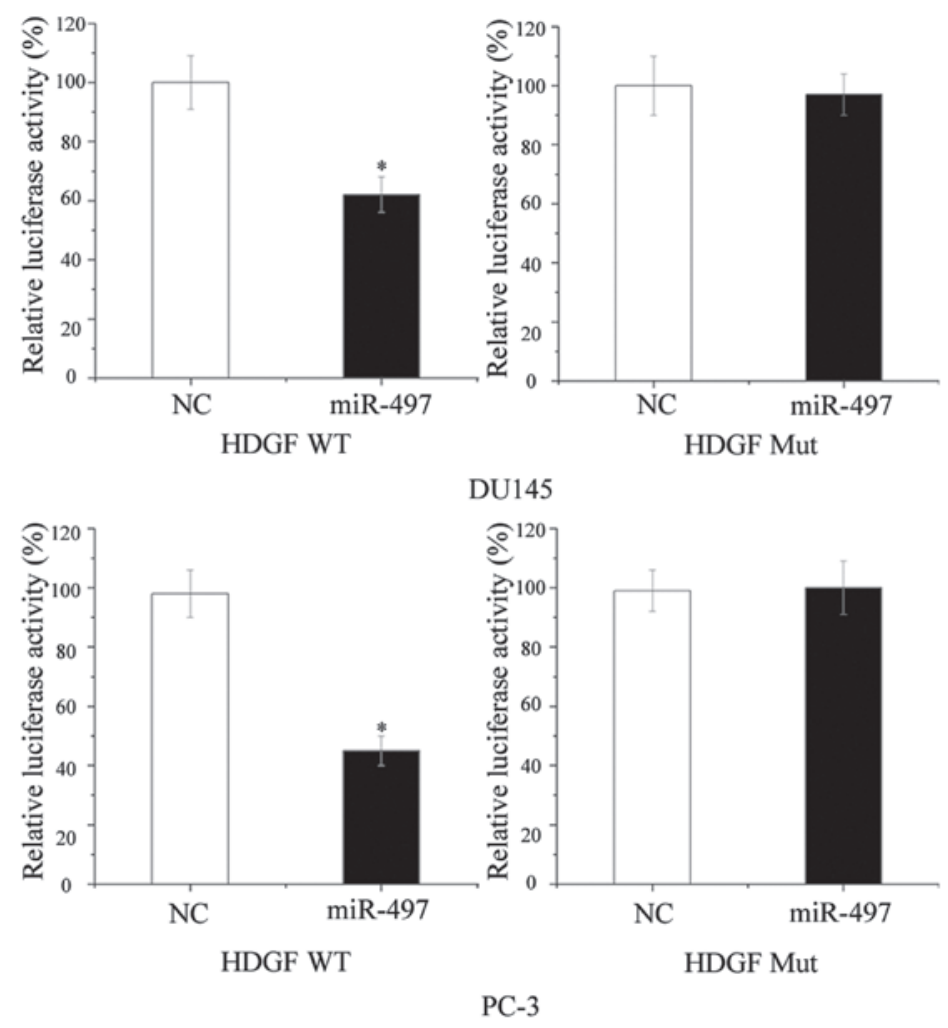

Figure 3. HDGF may be a direct target of miR-497. miR-497 reduced the activity of a HDGF 3'UTR-driven luciferase reporter vector in DU145 and PC-3 cells after transfection with miR-497. Values are expressed as the mean \pm standard deviation. " $\mathrm{P}<0.05$, compared with the control. UTR, untranslated region; miR, microRNA; NC, negative control; hsa, Homo sapiens; HDGF, hepatoma-derived growth factor; WT, wild-type; Mut, mutated.

and PC-3 cells were subjected to luciferase reporter assays. As illustrated in Fig. 3, transfection with miR-497 significantly decreased the activity of the HDGF 3'-UTR luciferase reporter compared with that in the NC-transfected group $(\mathrm{P}<0.05)$, while the activity of the reporter containing the mutated sequence was not affected. These results confirmed in vitro that HDGF was a target gene of miR-497 in prostate cancer cells.

\section{Discussion}

Metastasis is a major event associated with tumor recurrence and poor patient outcome (19). miRs are a group of small, non-coding RNAs that are commonly dysregulated in human malignant cancers (20). The involvement of miRs in the development of tumor metastasis has provided a novel perspective on the tumorigenic process (21). The present study revealed that miR-497 may function as a tumor suppressor through repression of HDGF in prostate cancer. Restoration of miR-497 suppressed cell migration and invasion in prostate cancer cells.

miR-195 and miR-497 are encoded by the same gene cluster located on chromosomal band 17p13.1, and due to their sequence AGCAGC starting at either the first nucleotide or the second nucleotide from the $5^{\prime}$ end of the mature $(\sim 22 \mathrm{nt}$, single stranded) miRNA, they are members of the miR-15/107 gene group (22). Expression of miR-497 has been proved to be frequently reduced in various tumors, including breast cancer (23), hepatocellular carcinoma (24), gastric cancer (25), nasopharyngeal carcinoma (26), endometrial cancer (27), colorectal cancer (28) and prostate cancer (18). In breast cancer, it has been demonstrated that miR-497 markedly inhibits cellular growth, induces apoptosis and disrupts the cell cycle by targeting Bcl-w; furthermore, miR-497 expression was negatively correlated with the pathological stage, lymphatic metastasis, tumor size and HER-2 (29). In hepatocellular carcinoma, it was demonstrated that CHEK1 is a target gene of miR-497 (24).

Identification of the target genes of miR-497 is crucial for understanding its role in tumorigenesis and for the discovery of novel therapeutic targets. Studies have certified that in human cells, miR-497 regulates the expression of certain oncogenes, including Bcl-w, ERK1, MEK1, IGF-1R, CHEK1 and MAPK (24,28-31). Therefore, upregulation of miR-497 or administration of pharmaceutical compounds with analogous effects is expected to represent an effective therapeutic strategy for tumors resulting from activation or overexpression of these oncogenes. The present study demonstrated the tumor suppressive effects of miR-497 in DU145 and PC-3 cell lines, which included the inhibition of cell migration and invasion by downregulation of HDGF expression. The findings of the present study suggested that miR-497 may represent a novel molecular marker and therapeutic agent to inhibit metastasis of prostate cancer.

HDGF is a novel growth factor with acidic heparin-binding ability and was first isolated from the cultured media of the HuH-7 human hepatoma cell line (32). It consists of 240 amino acids with functional domains, including a conserved N-terminal 100-amino-acid domain responsible for heparin and DNA binding, and two bipartite nuclear localization sequences (33). Studies have revealed that HDGF is 
expressed in most organs (i.e. liver, brain, lung and intestine) during embryonic development as well as in mature organisms (34). Besides its mitogenic activity, HDGF is implicated in organ development and tissue differentiation (35). HDGF has been found to be highly expressed in non-small cellular lung cancer (36), hepatocellular carcinoma (37), colorectal carcinoma (38), oesophagal carcinoma (39), pancreatic carcinoma (40) and melanoma (41). In addition, high levels of HDGF are associated with poor prognosis in several types of cancer, including non-small cell lung cancer, hepatocellular carcinoma, esophageal cancer, gastric cancer and gastrointestinal stromal tumors (33). Therefore, HDGF represents a potential prognostic factor for these cancers types.

The effects of HDGF overexpression in carcinogenesis have become a main focus in cancer biology and have been studied in various types of cancer. Previous studies have indicated that HDGF participates in numerous cellular processes, including astrocyte proliferation, renal development, vascular lesion formation (42), sensitization to irradiation (43), cardiovascular differentiation as well as cell migration and invasion (44-48). In prostate cancer, HDGF was shown to be overexpressed and knockdown of HDGF consistently inhibited proliferation. Moreover, knockdown of HDGF inhibited the migration and invasion of the DU145 prostate cancer cell line (48). The results of the present study suggested that miR-497 suppressed prostate cancer cell migration and invasion through downregulation of HDGF. miR-497 may therefore be utilized as a predictive parameter for early detection of tumor recurrence and as a target of drugs to block prostate cancer metastasis.

In conclusion, the present study was the first to show that miR-497 regulates HDGF and regulates cell migration and invasion of prostate cancer cells. The identification of candidate target genes of miR-497 may provide an understanding of potential carcinogenic mechanisms in prostate cancer. miRNA-based therapy is expected to be more efficient than the traditional single target therapy, since miRNAs regulate multiple target genes simultaneously. Therefore, miR-497 may be utilized for the treatment of prostate cancer.

\section{References}

1. Wu D, Huang $\mathrm{P}$, Wang $\mathrm{L}$, Zhou $\mathrm{Y}$, Pan $\mathrm{H}$ and $\mathrm{Qu} \mathrm{P}$ MicroRNA-143 inhibits cell migration and invasion by targeting matrix metalloproteinase 13 in prostate cancer. Mol Med Rep 8: 626-630, 2013.

2. Siegel R, Ma J, Zou Z and Jemal A: Cancer statistics, 2014. CA Cancer J Clin 64: 9-29, 2014

3. Mangoni M, Desideri I, Detti B, Bonomo P, Greto D, Paiar F, Simontacchi G, Meattini I, Scoccianti S, Masoni T, et al: Hypofractionation in prostate cancer: Radiobiological basis and clinical appliance. Biomed Res Int 2014: 781340, 2014

4. Lafeuille MH, Gravel J, Grittner A, Lefebvre P, Ellis L and McKenzie RS: Real-World corticosteroid utilization patterns in patients with metastatic castration-resistant prostate cancer in 2 large US administrative claims databases. Am Health Drug Benefits 6: 307-316, 2013.

5. Nelson CJ, Lee JS, Gamboa MC and Roth AJ: Cognitive effects of hormone therapy in men with prostate cancer: A review. Cancer 113: 1097-1106, 2008.

6. Discacciati A and Wolk A: Lifestyle and dietary factors in prostate cancer prevention. Recent Results Cancer Res 202: 27-37, 2014.

7. Lai GY, Giovannucci EL, Pollak MN, Peskoe SB, Stampfer MJ, Willett WC and Platz EA: Association of C-peptide and leptin with prostate cancer incidence in the health professionals follow-up study. Cancer Causes Control 25: 625-632, 2014.
8. $\mathrm{Wu} \mathrm{J}$ and $\mathrm{Yu} \mathrm{E}$ : Insulin-like growth factor receptor-1 (IGF-IR) as a target for prostate cancer therapy. Cancer Metastasis Rev 33: 607-617, 2014.

9. Barba M, McCann SE, Schünemann HJ, Stranges S, Fuhrman B, De Placido S, Carruba G, Freudenheim JL, Trevisan M, Russell M, et al: Lifetime total and beverage specific-alcohol intake and prostate cancer risk: A case-control study. Nutr J 3: 23,2004

10. Joung JG, Kim D, Lee SY, Kang HJ and Kim JH: Integrated analysis of microRNA-target interactions with clinical outcomes for cancers. BMC Med Genomics 7 (Suppl 1): S10, 2014.

11. Garzon R, Pichiorri F, Palumbo T, Visentini M, Aqeilan R, Cimmino A, Wang H, Sun H, Volinia S, Alder H, et al: MicroRNA gene expression during retinoic acid-induced differentiation of human acute promyelocytic leukemia. Oncogene 26: 4148-4157, 2007.

12. Carthew RW and Sontheimer EJ: Origins and Mechanisms of miRNAs and siRNAs. Cell 136: 642-655, 2009.

13. Friedman RC, Farh KK, Burge CB and Bartel DP: Most mammalian mRNAs are conserved targets of microRNAs. Genome Res 19: 92-105, 2009.

14. Kwak PB, Iwasaki S and Tomari Y: The microRNA pathway and cancer. Cancer Sci 101: 2309-2315, 2010.

15. Oom AL, Humphries BA and Yang C: MicroRNAs: Novel players in cancer diagnosis and therapies. Biomed Res Int 2014: 959461, 2014

16. Esquela-Kerscher A and Slack FJ: Oncomirs-microRNAs with a role in cancer. Nat Rev Cancer 6: 259-269, 2006.

17. Calin GA and Croce CM: MicroRNA signatures in human cancers. Nat Rev Cancer 6: 857-866, 2006.

18. Wang L, Li B, Li L and Wang T: MicroRNA-497 suppresses proliferation and induces apoptosis in prostate cancer cells. Asian Pac J Cancer Prev 14: 3499-3502, 2013.

19. Amate $P$, Huchon C, Dessapt AL, Bensaid C, Medioni J, Le Frère Belda MA, Bats AS and Lécuru FR: Ovarian cancer: SItes of recurrence. Int J Gynecol Cancer 23: 1590-1596, 2013.

20. Martignani E, Miretti S, Accornero P and Baratta M: MiRNAs highlights in stem and cancer cells. Mini Rev Med Chem 11: 1165-1182, 2011.

21. Hummel R, Hussey DJ and Haier J: MicroRNAs: Predictors and modifiers of chemo- and radiotherapy in different tumour types. Eur J Cancer 46: 298-311, 2010.

22. Finnerty JR, Wang WX, Hébert SS, Wilfred BR, Mao G and Nelson PT: The miR-15/107 group of microRNA genes: Evolutionary biology, cellular functions and roles in human diseases. J Mol Biol 402: 491-509, 2010.

23. Li D, Zhao Y, Liu C, Chen X, Qi Y, Jiang Y, Zou C, Zhang X, Liu S, Wang X, et al: Analysis of MiR-195 and MiR-497 expression, regulation and role in breast cancer. Clin Cancer Res 17: 1722-1730, 2011

24. Xie Y, Wei RR, Huang GL, Zhang MY, Yuan YF and Wang HY: Checkpoint kinase 1 is negatively regulated by miR-497 in hepatocellular carcinoma. Med Oncol 31: 844, 2014.

25. Guo J, Miao Y, Xiao B, Huan R, Jiang Z, Meng D and Wang Y: Differential expression of microRNA species in human gastric cancer versus non-tumorous tissues. J Gastroenterol Hepatol 24: 652-657, 2009.

26. Chen HC, Chen GH, Chen YH, Liao WL, Liu CY, Chang KP, Chang YS and Chen SJ: MicroRNA deregulation and pathway alterations in nasopharyngeal carcinoma. Br J Cancer 100: 1002-1011, 2009.

27. Hiroki E, Akahira J, Suzuki F, Nagase S, Ito K, Suzuki T, Sasano $\mathrm{H}$ and Yaegashi N: Changes in microRNA expression levels correlate with clinicopathological features and prognoses in endometrial serous adenocarcinomas. Cancer Sci 101: 241-249, 2010.

28. Guo ST, Jiang CC, Wang GP, Li YP, Wang CY, Guo XY, Yang RH, Feng Y, Wang FH, Tseng HY, et al: MicroRNA-497 targets insulin-like growth factor 1 receptor and has a tumour suppressive role in human colorectal cancer. Oncogene 32: 1910-1920, 2013

29. Shen L, Li J, Xu L, Ma J, Li H, Xiao X, Zhao J and Fang L: MiR-497 induces apoptosis of breast cancer cells by targeting Bcl-w. Exp Ther Med 3: 475-480, 2012.

30. Zheng D, Radziszewska A and Woo P: MicroRNA 497 modulates interleukin 1 signalling via the MAPK/ERK pathway. FEBS Lett 586: 4165-4172, 2012.

31. Luo M, Shen D, Zhou X, Chen X and Wang W: MicroRNA-497 is a potential prognostic marker in human cervical cancer and functions as a tumor suppressor by targeting the insulin-like growth factor 1 receptor. Surgery 153: 836-847, 2013. 
32. Chen X, Yun J, Fei F, Yi J, Tian R, Li S and Gan X: Prognostic value of nuclear hepatoma-derived growth factor (HDGF) localization in patients with breast cancer. Pathol Res Pract 208: 437-443, 2012

33. Lin YW, Li CF, Chen HY, Yen CY, Lin LC, Huang CC, Huang HY, Wu PC, Chen $\mathrm{CH}$, Chen SC and Tai MH: The expression and prognostic significance of hepatoma-derived growth factor in oral cancer. Oral Oncol 48: 629-635, 2012.

34. Abouzied MM, Baader SL, Dietz F, Kappler J, Gieselmann V and Franken S: Expression patterns and different subcellular localization of the growth factors HDGF (hepatoma-derived growth factor) and HRP-3 (HDGF-related protein-3) suggest functions in addition to their mitogenic activity. Biochem J 378: 169-176, 2004.

35. Sedlmaier A, Wernert N, Gallitzendörfer R, Abouzied MM, Gieselmann V and Franken S: Overexpression of hepatoma-derived growth factor in melanocytes does not lead to oncogenic transformation. BMC Cancer 11: 457, 2011.

36. Ren H, Tang X, Lee JJ, Feng L, Everett AD, Hong WK, Khuri FR and Mao L: Expression of hepatoma-derived growth factor is a strong prognostic predictor for patients with early-stage non-small-cell lung cancer. J Clin Oncol 22: 3230-3237, 2004.

37. Hu TH, Huang CC, Liu LF, Lin PR, Liu SY, Chang HW, Changchien CS, Lee CM, Chuang JH and Tai MH: Expression of hepatoma-derived growth factor in hepatocellular carcinoma. Cancer 98: 1444-1456, 2003.

38. Lepourcelet M, Tou L, Cai L, Sawada J, Lazar AJ, Glickman JN, Williamson JA, Everett AD, Redston M, Fox EA, et al: Insights into developmental mechanisms and cancers in the mammalian intestine derived from serial analysis of gene expression and study of the hepatoma-derived growth factor (HDGF). Development 132: 415-427, 2005.

39. Yamamoto S, Makuuchi H, Shimada H, Chino O, Nishi T, Kise Y, Kenmochi T and Hara T: Clinical analysis of reflux esophagitis following esophagectomy with gastric tube reconstruction. J Gastroenterol 42: 342-345, 2007.
40. Uyama H, Tomita Y, Nakamura H, Nakamori S, Zhang B, Hoshida Y, Enomoto H, Okuda Y, Sakon M, Aozasa K, et al: Hepatoma-derived growth factor is a novel prognostic factor for patients with pancreatic cancer. Clin Cancer Res 12: 6043-6048, 2006.

41. Bernard K, Litman E, Fitzpatrick JL, Shellman YG, Argast G, Polvinen K, Everett AD, Fukasawa K, Norris DA, Ahn NG and Resing KA: Functional proteomic analysis of melanoma progression. Cancer Res 63: 6716-6725, 2003.

42. Okuda Y, Nakamura H, Yoshida K, Enomoto H, Uyama H, Hirotani T, Funamoto M, Ito H, Everett AD and Hada T: Hepatoma-derived growth factor induces tumorigenesis in vivo through both direct angiogenic activity and induction of vascular endothelial growth factor. Cancer Sci 94: 1034-1041, 2003.

43. Matsuyama A, Inoue H, Shibuta K, Tanaka Y, Barnard GF, Sugimachi K and Mori M: Hepatoma-derived growth factor is associated with reduced sensitivity to irradiation in esophageal cancer. Cancer Res 61: 5714-5717, 2001.

44. Everett AD: Identification, cloning and developmental expression of hepatoma-derived growth factor in the developing rat heart. Dev Dyn 222: 450-458, 2001.

45. Everett AD, Lobe DR, Matsumura ME, Nakamura $H$ and McNamara CA: Hepatoma-derived growth factor stimulates smooth muscle cell growth and is expressed in vascular development. J Clin Invest 105: 567-575, 2000.

46. Oliver JA and Al-Awqati Q: An endothelial growth factor involved in rat renal development. J Clin Invest 102: 1208-1219, 1998.

47. Crossin KL, Tai MH, Krushel LA, Mauro VP and Edelman GM: Glucocorticoid receptor pathways are involved in the inhibition of astrocyte proliferation. Proc Natl Acad Sci USA 94: 2687-2692, 1997.

48. Guo Z, He Y, Wang S, Zhang A, Zhao P, Gao C and Cao B: Various effects of hepatoma-derived growth factor on cell growth, migration and invasion of breast cancer and prostate cancer cells. Oncol Rep 26: 511-517, 2011. 